\title{
TEMPERATURE DISTRIBUTION AND REACTION CHARACTERISTICS OF THE HORIZONTAL EPITAXIAL REACTOR IN HIGH FLOW RATE RANGE
}

\author{
TAMOTSU HANZAWA, Kunio KATO AND TeIRIKI TADAKI \\ Department of Chemical Engineering, Gunma University, \\ Kiryu 376
}

\begin{abstract}
The temperature distributions and concentration distributions of reactant gas in a horizontal epitaxial reactor were investigated experimentally and theoretically in the high flow rate range, that is, $Q=10-60 \mathrm{l} / \mathrm{min}\left(20^{\circ} \mathrm{C}, 1 \mathrm{~atm}, R e=160-1400\right)$. The temperature and concentration distributions in the reactor in the case of no axial temperature gradient in the high flow rate range were between the calculated distributions for spiral flow and the calculated distributions for axial flow with no cross-sectional flow. When the flow rate is less than $20 \mathrm{l} / \mathrm{min}$, the temperature and concentration distributions are approximately calculated by the fundamental equations in the case of stable spiral flow. However, when the flow rate is larger than $50 \mathrm{l} / \mathrm{min}$, these distributions are approximately calculated by the fundamental equations in the case of axial flow with no crosssectional flow. An empirical equation for the estimation of gas temperature distributions in the high flow rate range was obtained.
\end{abstract}

\section{Introduction}

In the epitaxial reactor (chemical vapor deposition reactor), deposition reaction occurs on the surface of a water. The thickness of reaction products, which must be as strictly uniform as possible in the case of production of semiconductor materials, are influenced by the temperature distribution and the concentration distribution of reactants in the reactor. Recently, transport phenomena in the horizontal epitaxial reactor were analyzed theoretically for the case of spiral flow. The calculated velocity and temperature distributions and the concentration distribution of a reactant gas agreed well with experimental ones ${ }^{2-4)}$.

Usually, two stable symmetrical spiral flows were generated in the reactor in the case of low Reynolds number. However, when the flow rate is increased, the spiral flow gradually degrades. The fluid flow pattern in the reactor was estimated approximately by Reynolds number. If $R e$ was larger than 80 , the spiral flow was gradually disturbed and when $R e$ was larger than 190 , no spiral flow was generated ${ }^{11}$.

Fluid flow pattern in the horizontal epitaxial reactor, where $R e>190$, became a laminar flow with small cross-sectional velocity components. The flow rate range where the steady spiral flow disappears is defined

Received September 12, 1980. Correspondence concerning this article should be addressed to T. anzawa. T. Tadaki is at Dept. of Chem. Eng., Tohoku Univ., Sendai 980. as the high flow rate range. Usually, industrial epitaxial reactors are operated not only in the spiral flow range but also in the high flow rate range. Therefore, it is very important to analyze transport phenomena in the high flow rate range. However, there have been few theoretical studies of transport phenomena in this range up to this time. To obtain the temperature distributions in the reactor in the high flow rate range, the cross-sectional and axial temperature distributions in the reactor were measured and the empirical equation for their estimation was obtained. To investigate the reaction characteristics, carbon plates in the reactor were burned and the local concentration of carbon dioxide was measured.

Furthermore, the temperature distribution and the concentration distribution of reactant gas were calculated numerically both from fundamental equations based upon the stable spiral flows and from equations based upon axial flow with no cross-sectional flow, and these results were compared with experimental ones.

\section{Experimental Apparatus and Procedure}

1. 1 Measurement of temperature distribution in the reactor

Figure 1 (a) is a schematic diagram of the experimental apparatus for the measurement of temperature distributions. Circular tubes with diameters of 5.0, $6.6,8.3 \mathrm{~cm}$ and rectangular channels with cross-sectional dimensions of $2.6 \times 4.3 \mathrm{~cm}$ and $1.7 \times 4.3 \mathrm{~cm}$ 


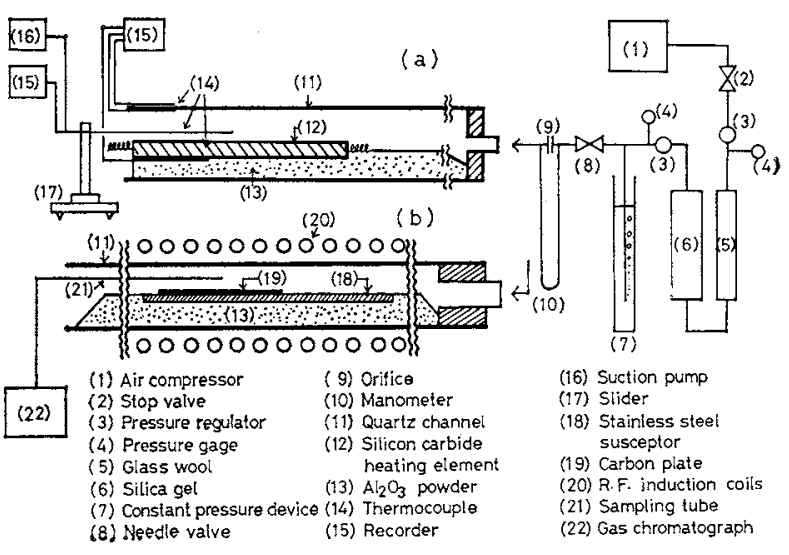

Fig. 1 Schematic diagram of experimental apparatus

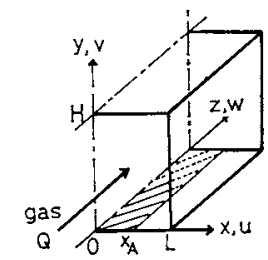

Fig. 2 Coordinate system for a horizontal rectangular channel

made of transparent quartz glass were used in this experiment. Length of these tubes was $300 \mathrm{~cm}$. A silicon carbide heating element $(2 \mathrm{~cm} \phi)$ covered by porcelain tube, in which Pt-PtRh thermocouples $(0.3 \mathrm{~mm} \phi)$ were fitted, was placed on the bottom of the reactor. $\mathrm{Al}_{2} \mathrm{O}_{3}$ powder was packed around the circumference of the heating element for heat insulation.

A suction pyrometer was used to measure the gas temperature. Pt-PtRh thermocouples $(0.3 \mathrm{~mm} \phi)$ were inserted into this pyrometer, and suction rate was adjusted so as not to disturb the flow pattern in the reactor. Purified air was used as feed gas. The gas flow rates of air were in the range of 10 to $60 \mathrm{l} / \mathrm{min}$ (at $20^{\circ} \mathrm{C}, 1 \mathrm{~atm}, R e=160-1400$ ). (There was no spiral flow in these flow rates ${ }^{1}$.) Surface temperatures of the heating element were in the range of 600 to $1000^{\circ} \mathrm{C}$. The experimental procedure was almost the same as that described in the previous paper ${ }^{2}$.

\section{2 Measurement of concentration distribution in the reactor}

To check the reaction characteristics in this reactor at high flow rate, carbon plates were burned on a susceptor and local concentrations of carbon dioxide were measured.

Figure 1 (b) shows a schematic diagram of the experimental apparatus for the measurement of concentration distribution. Two rectangular channels, with cross-sectional dimensions of $1.7 \times 4.3$ and $2.6 \times$ $4.3 \mathrm{~cm}$ and made of transparent quartz glass, were used in this experiment. The length of these tubes was equal to that used for the measurement of temperature distribution. A stainless steel susceptor $\left(2.0 \times 1.0 \times 130 \mathrm{~cm}^{3}\right)$ was placed on the bottom of the channel and was heated by RF induction coil. A carbon plate was put on the middle of this susceptor to establish the temperature and velocity distributions of the reactant gas in the axial direction. The carbon plate was graphitic carbon SEG-5 made by Nippon Carbon Co., Ltd. and was burned at $750^{\circ} \mathrm{C}$ where the overall reaction rate was controlled by the surface reaction rate. Purified air was used as reactant gas and the flow rates of air were the same as those in the case of temperature measurement.

The experimental procedure was almost the same as that described in the previous paper ${ }^{3,4)}$.

\section{Fundamental Equations and Numerical Calculation Procedure}

To analyze the temperature and reactant concentration distributions in the horizontal epitaxial reactor in the high flow rate range, two cases are considered: three-dimensional flow with laminar natural convection in the cross-sectional direction (stable spiral flow) and axial forced convection flow with no natural convection. Figure 2 shows the coordinate system for this calculation.

For the fundamental differential equations in threedimensional flow with laminar natural convection, the following assumptions are made from the observed flow pattern in this reactor.

1) Since the reactant gas is well preheated before entering the reaction zone, temperature distribution and flow pattern in the reactor are established and are not affected by its axial length.

2) Since $P e$ is quite large for the reactor, the axial conduction and diffusion term in the energy and mass balance equations may be neglected.

3) The physical properties of fluid, such as viscosity, thermal conductivity, etc., are independent of temperature, but the buoyancy force is dependent upon the temperature difference.

From the above assumptions, the fundamental equations for the continuity, momentum, energy and mass balance and the boundary conditions can be written in dimensionless form as follows:

$$
\begin{gathered}
\frac{\partial U}{\partial X}+\frac{\partial V}{\partial Y}=0 \\
U \frac{\partial U}{\partial X}+V \frac{\partial U}{\partial Y}+\frac{\partial P}{\partial X}=\frac{\partial^{2} U}{\partial X^{2}}+\frac{\partial^{2} U}{\partial Y^{2}} \\
U \frac{\partial V}{\partial X}+V \frac{\partial V}{\partial Y}+\frac{\partial P}{\partial Y}=\frac{\partial^{2} V}{\partial X^{2}}+\frac{\partial^{2} V}{\partial Y^{2}}-G r T \\
U \frac{\partial W}{\partial X}+V \frac{\partial W}{\partial Y}+\frac{\partial P}{\partial Z}=\frac{\partial^{2} W}{\partial X^{2}}+\frac{\partial^{2} W}{\partial Y^{2}}
\end{gathered}
$$




$$
\begin{aligned}
& U \frac{\partial T}{\partial X}+V \frac{\partial T}{\partial Y}=\frac{1}{P r}\left(\frac{\partial^{2} T}{\partial X^{2}}+\frac{\partial^{2} T}{\partial Y^{2}}\right) \\
& U \frac{\partial C}{\partial X}+V \frac{\partial C}{\partial Y}+W \frac{\partial C}{\partial Z}=\frac{1}{S c}\left(\frac{\partial^{2} C}{\partial X^{2}}+\frac{\partial^{2} C}{\partial Y^{2}}\right)
\end{aligned}
$$

(B.C)

$$
\begin{aligned}
& X=0 ; U=\frac{\partial V}{\partial X}=\frac{\partial W}{\partial X}=\frac{\partial T}{\partial X}=\frac{\partial C}{\partial X}=0 \\
& X=\frac{L}{H} ; U=V=W=T=\frac{\partial C}{\partial X}=0 \\
& Y=0 ;\left\{\begin{array}{l}
0 \leqq X \leqq \frac{X_{A}}{H} ; U=V=W=0 \\
T=1, \mathscr{D} \frac{\partial C}{\partial Y}=K C_{H} \\
\frac{X_{A}}{H}<X \leqq \frac{L}{H} ; U=V=W=T=\frac{\partial C}{\partial Y}=0
\end{array}\right. \\
& Y=1 ; U=V=W=T=\frac{\partial C}{\partial Y}=0 \\
& Z=0 ; C=1
\end{aligned}
$$

The dimensionless stream function $\Psi$ and the vorticity $\zeta$ are defined and after some manipulations the nondimensional fundamental equations were solved numerically with the boundary conditions. The calculation procedure is the same as those in the previous paper $^{2-4)}$.

To calculate the temperature and reactant concentration distribution in the case of axial forced convective flow with no natural convection, from the above assumptions (2) and (3) the fundamental equations and the boundary conditions can be written in dimensionless forms as follows:

$$
\begin{aligned}
\frac{\partial P}{\partial Z} & =\frac{\partial^{2} W}{\partial X^{2}}+\frac{\partial^{2} W}{\partial Y^{2}} \\
W \frac{\partial T}{\partial Z} & =\frac{1}{P r}\left(\frac{\partial^{2} T}{\partial X^{2}}+\frac{\partial^{2} T}{\partial Y^{2}}\right) \\
W \frac{\partial C}{\partial Z} & =\frac{1}{S c}\left(\frac{\partial^{2} C}{\partial X^{2}}+\frac{\partial^{2} C}{\partial Y^{2}}\right)
\end{aligned}
$$

(B.C) In addition to Eq. (7),

$$
Z=0 ; W=F(X, Y), T=0, C=1
$$

Equations (7)-(11) were solved numerically as follows: At first each equation was written in a finite difference form. Then, specifying the axial pressure gradient from the flow rate of gas, the axial velocity component, $W$, was calculated from Eqs. (7) and (8) with the relaxation method as a function of $X$ and $Y$. Finally, the temperature and concentration distributions were calculated from Eqs. (7), (9), (10) and (11) by using this velocity.

\section{Experimental Results and Comparison of Calculated with Experimental Results}

\section{1 Temperature distribution}

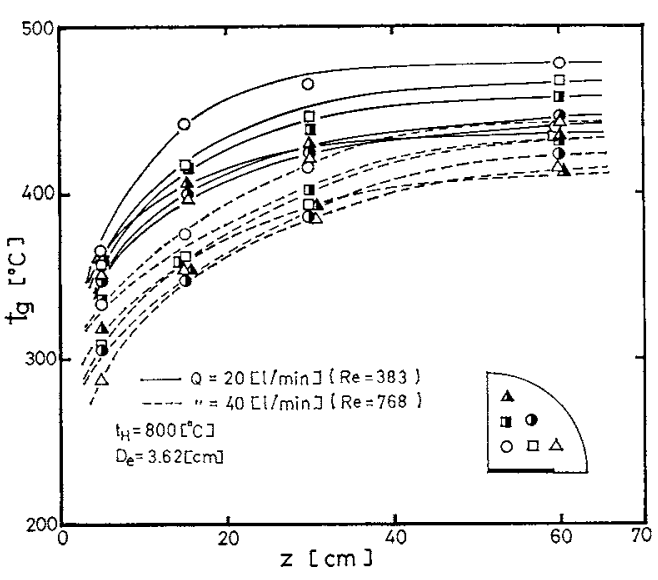

Fig. 3 Relation between local temperature distributions and axial distance for $Q=20$ and $40 \mathrm{l} / \mathrm{min}$ with experimental point as parameter

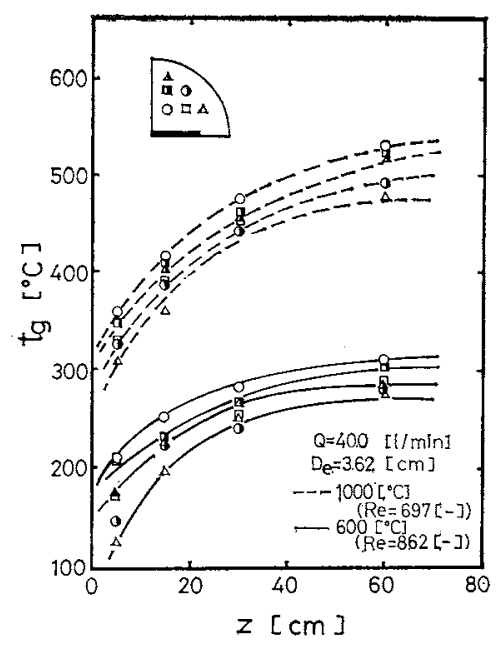

Fig. 4 Relation between local temperature distributions and axial distance for $t_{H}=600$ and $1000^{\circ} \mathrm{C}$

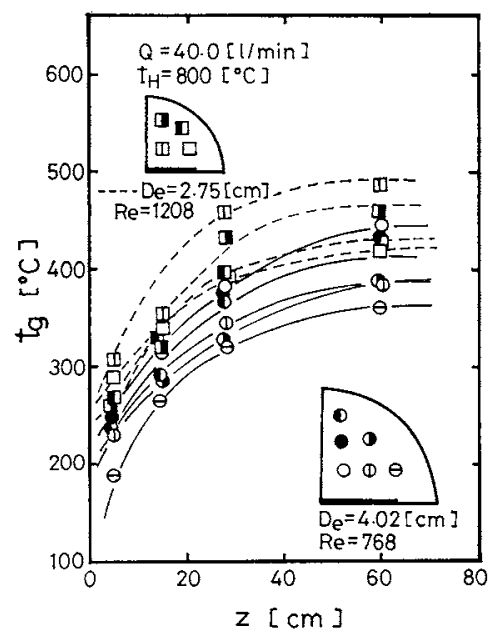

Fig. 5 Relation between local temperature distributions and axial distance for $D_{\varepsilon}=2.75$ and $4.02 \mathrm{~cm}$

Some typical axial gas temperature distributions for several operating conditions are shown in Figs. 3-5. 

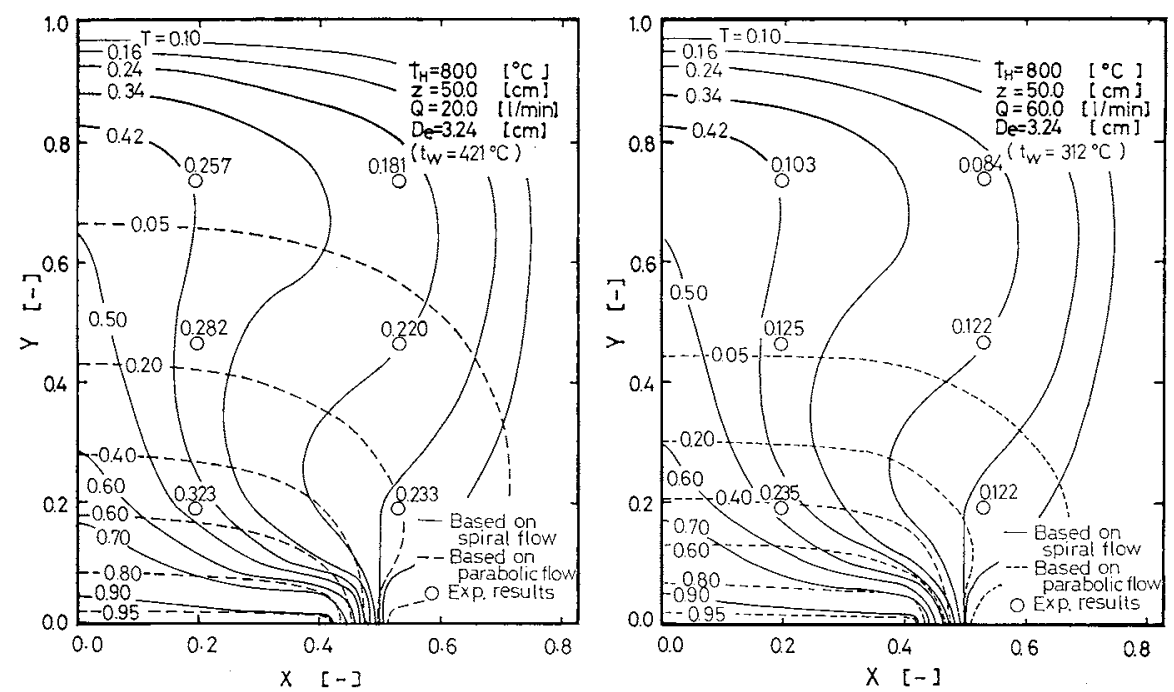

Fig. 6 Temperature distributions with $Q=20$ and $60 \mathrm{l} / \mathrm{min}(R e=315$ and 951$)$ for $L / H=0.827$, $D_{e}=3.24 \mathrm{~cm}, x_{A} / H=0.415, G r=2.72 \times 10^{4}$ and $P r=0.69$
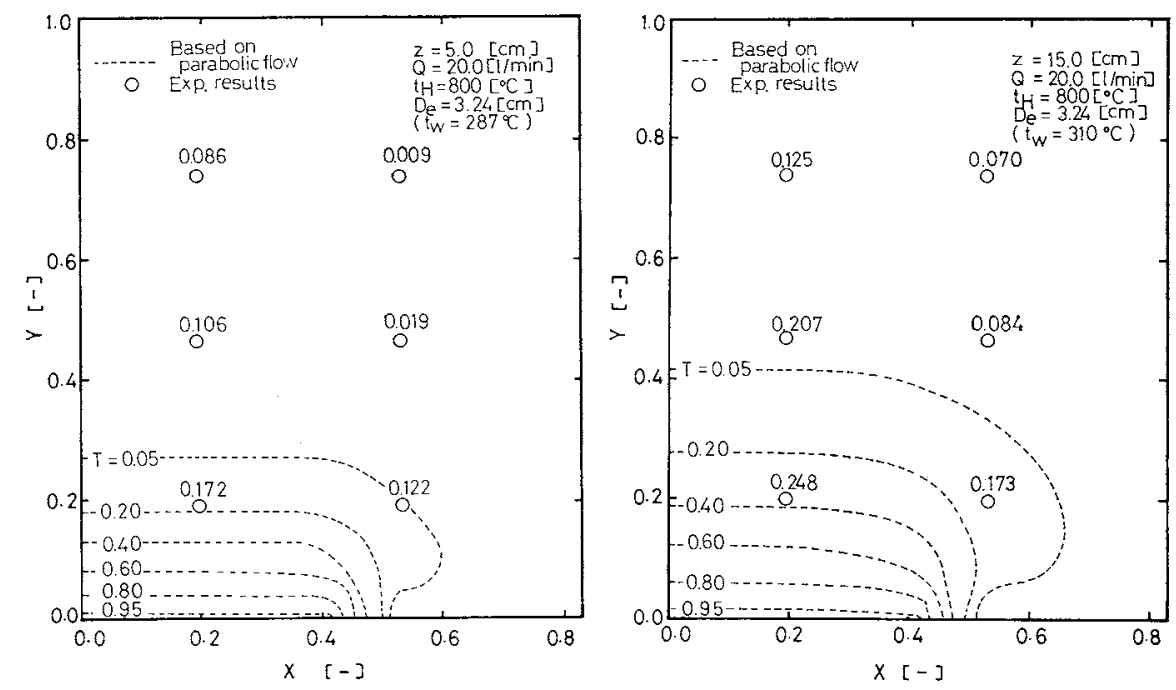

Fig. 7 Temperature distributions with $z=5$ and $15 \mathrm{~cm}$ for $L / H=0.827$, $D_{e}=3.24 \mathrm{~cm}$ and $x_{A} / H=0.415$

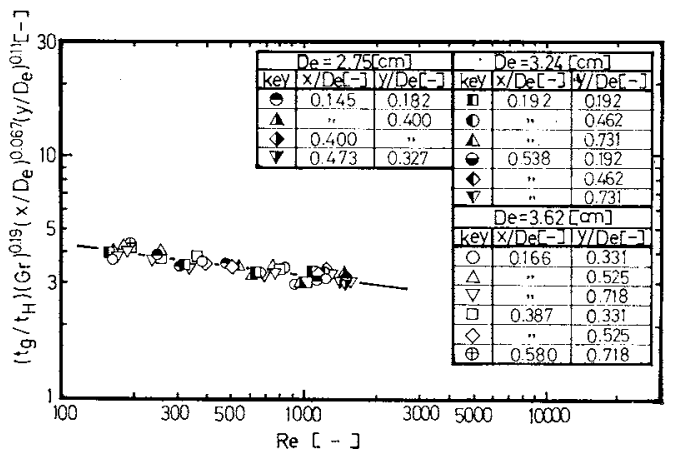

Fig. 8 Correlation between $\operatorname{Re}$ and $\left(t_{g} / t_{H}\right)(G r)^{0.10}$. $\left(\boldsymbol{x} / \boldsymbol{D}_{e}\right)^{0.067}\left(\boldsymbol{y} / \boldsymbol{D}_{e}\right)^{0.11}$

Figure 3 shows the relationship between the local temperature distributions in the reactor and the axial distance from the leading edge of the heating element in the case of $Q=20$ and $40 \mathrm{l} / \mathrm{min}\left(20^{\circ} \mathrm{C}, 1 \mathrm{~atm}\right)$. From Fig. 3, it is found that when the flow rate of air is small, the axial temperature gradient becomes large and the thermal entrance region consequently becomes short.

Figure 4 shows the axial local temperature distributions in the case of $Q=40 \mathrm{l} / \mathrm{min}$ with the temperature of heating element as a parameter. When $t_{H}$ is increased, the temperature in the reactor becomes high and the thermal entrance region becomes long.

Figure 5 shows the axial local temperature distributions in the case of $Q=40 \mathrm{l} / \mathrm{min}$ and $t_{H}=800^{\circ} \mathrm{C}$ with equivalent diameter of tube, $D_{e}$, as a parameter. From this figure, it seems that when $D_{e}$ is small, the temperature in the reactor becomes high and the thermal entrance region becomes short.

Some typical experimental and calculated dimen- 

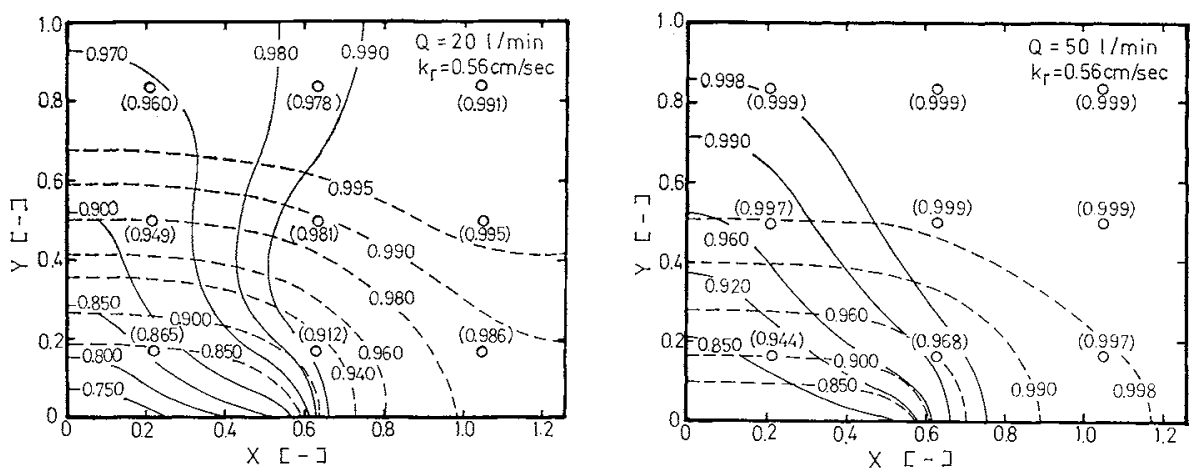

Fig. 9 Concentration distributions with $Q=20$ and $50 \mathrm{l} / \mathrm{min}(R e=374$ and 1040$)$ for $L / H=1.265$, $x_{A} / H=0.588, G r=1.1 \times 10^{4}, P r=0.69$ and $S c=0.63$

sionless temperature distributions in the cross-section are shown in Figs. 6 and 7. Since velocity and temperature distributions are symmetrical about the $Y$ axis in the reactor, these distributions are shown in the right-hand half of the cross-section. In Figs. 6 and 7 , the numerical values written near the experimental points are the dimensionless local temperatures obtained in this experiment. The solid lines show the temperature distributions calculated from the fundamental equations in the case of stable spiral flow and the dotted lines show the calculated values in the case of axial flow with no cross-sectional flow.

Figure 6 shows the temperature distributions at a distance of $50 \mathrm{~cm}$ from the leading edge of the heating element in the case of $Q=20$ and $60 \mathrm{l} / \mathrm{min}$, respectively. When the flow rate of air is small, the gas temperature in the reactor becomes high. From Fig. 6 , when the flow rate is smaller than $20 \mathrm{l} / \mathrm{min}$, the experimental values agree approximately with the calculated ones for stable spiral flow. However, when the flow rate is increased, the experimental values gradually approach the values for of axial velocity with no cross-sectional flow.

Figure 7 shows the effect of axial distance from the leading edge of the heating element upon the local temperature distribution in the reactor. From Figs. 6 and 7 , when the axial distance becomes long, the temperature in the reactor becomes high.

Generally, the temperature distribution in the thermal entrance region is higher than that calculated from the fundamental equations in the case of axial flow with no cross-sectional flow, as shown in Fig. 7. When the axial distance from the leading edge of the heating element becomes long, the temperature distribution gradually approaches the calculated one for stable spiral flow in low flow rate range $(Q \leqq 20 \mathrm{l} / \mathrm{min})$. 3. 2 Empirical equation of gas temperature distribution in the reactor

From Figs. 3 to 5, gas temperature in the horizontal epitaxial reactor is increased with increase of axial distance from the leading edge of the heating element. Finally, there is a place where no axial temperature gradient exists. The thermally established region in this reactor is defined as the place where no axial temperature gradient exists. From Fig. 6, when the flow rate of air is about 20 to $50 \mathrm{l} / \mathrm{min}$, gas temperature distributions in the thermally established region are intermediate between the calculated values for stable spiral flow and the calculated ones for axial flow with no cross-sectional flow. However, since gas temperature distribution in the thermally established region is difficult to estimate from the hydrodynamic calculation, the empirical equation for its estimation is obtained.

Gas temperatures in the thermally established region are affected by the following factors: $t_{H}, G r,\left(x / D_{e}\right)$, $\left(y / D_{e}\right)$ and $R e$. From the experimental data, it was found that $\left(t_{g} / t_{H}\right)$ is proportional to $\left(x / D_{e}\right)^{-0.067}$, $\left(y / D_{e}\right)^{-0.11}$ and $G r^{-0.19}$. Figure 8 shows the relationship between $R e$ and $\left(t_{g} / t_{H}\right)(G r)^{0.19}\left(x / D_{e}\right)^{0.067}\left(y / D_{e}\right)^{0.11}$. From Fig. 8, the following empirical equation for the estimation of temperature distributions in the thermal established region is obtained.

$$
\left(t_{g} / t_{Y H}\right)=7.789(R e)^{-0.128}(G r)^{-0.19}\left(x / D_{e}\right)^{-0.067}\left(y / D_{e}\right)^{-0.11}
$$

Equation (12) is applicable when $R e$ is $160-1400, G r$ is $8 \times 10^{3}-5 \times 10^{4}, x / D_{e}$ is $0.1-0.7$ and $y / D_{e}$ is $0.1-$ 0.7 and Eq. (12) is applied to circular tubes and rectangular channels.

\section{3 Concentration distribution of reactant gas}

Figure 9 shows dimensionless concentration distributions of the reactant gas in the cross-section at a distance of $20 \mathrm{~cm}$ from the leading edge of the carbon plate in the case where the flow rates of feed air are 20 and $50 \mathrm{l} / \mathrm{min}$, respectively.

In Fig. 9 the solid and dotted lines and the numerical values written near the experimental points are in the same situation as those in Figs. 6 and 7. From Fig. 9 , the experimental values agree approximately 


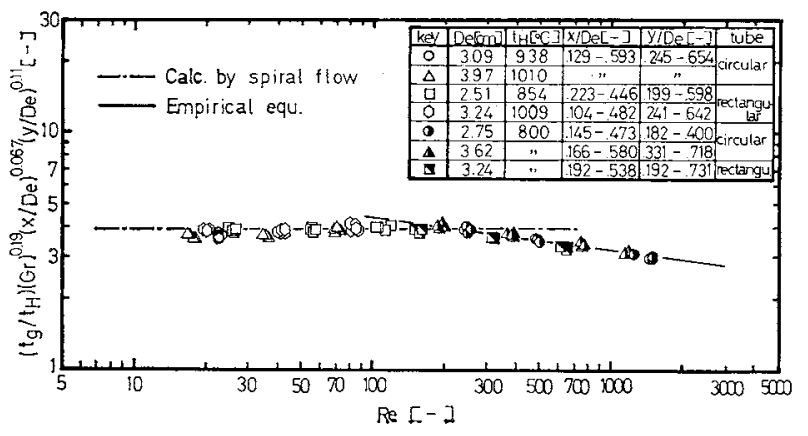

Fig. 10 Relation between temperature in thermal established region and $R e$

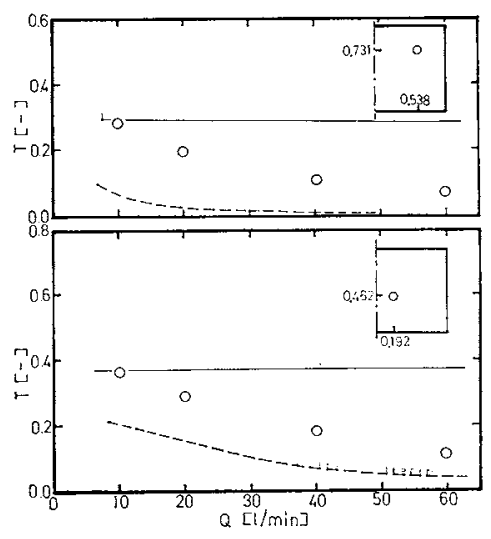

(a) Temperature $\left(G r=2.66 \times 10^{4}-2.81 \times 10^{4}\right.$, $L / H=0.827, \quad x_{A} / H=0.415, \quad D_{e}=3.24$, $t_{H}=800^{\circ} \mathrm{C}$ and $\left.z=50 \mathrm{~cm}\right)$

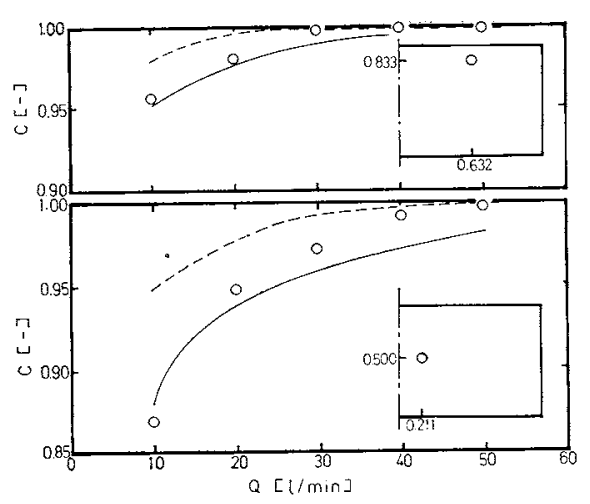

(b) Concentration $\left(G r=1.1 \times 10^{4}, L / H=\right.$ $1.265, x_{A} / H=0.588$ and $k_{r}=0.56 \mathrm{~cm} /$ sec)

_ : calc. by spiral flow

_. : calc. by parabolic flow

$\bigcirc$ : exp. results

Fig. 11 Comparison of calculated temperature and concentration with experimental ones

with the calculated ones based upon stable spiral flow where the flow rate is less than $20 \mathrm{l} / \mathrm{min}$. However, when the flow rate is increased, the measured reactant concentrations gradually approached the calculated ones for axial flow with no cross-sectional flow.

\section{Discussion}

Temperature distribution in the reactor was also investigated in the stable spiral flow range ${ }^{2)}$. Figure 10 shows the relationship between temperature in the reactor in the case of the thermal established region and Reynolds number. The dot-dash line in Fig. 10 shows the calculated value from the fundamental equations based upon steady spiral flow. The solid line shows the empirical Eq. (12) obtained in this study.

From Fig. 10, in the steady spiral flow range, the calculated values agree very well with the experimental ones. In the steady spiral flow range ( $R e \leqq 190)$, gas temperature in the reactor is not affected by the Reynolds number. However, in the high flow rate range, the gas temperature decreases gradually with increase of Reynolds number. This fact means that in the spiral flow range, the heat is transferred in the cross-sectional direction by natural convection and average gas temperature in the reactor increases. However, when the gas flow rate increases, the effect of natural convection on the heat transfer decreases relatively and average gas temperature decreases with increase of gas velocity.

The solid line (Eq. (12)) intersects the dot-dash line at about $R e=200$. This fact supports the result of the previous paper ${ }^{11}$, that when the Reynolds number is larger than 190 no spiral flow is generated in the reactor.

Generally speaking, when the flow rate increases, the concentration distribution of the reactant and gas temperature distribution in the reactor approach the calculated values based upon axial flow with no crosssectional flow. Figures 11 (a) and 11 (b) show the relationship between local gas temperature and flow rate, and that between local reactant concentration and flow rate, respectively.

From Fig. 11, experimental reactant concentration and gas temperature in the reactor agree approximately with calculated values based upon spiral flow in the case where gas flow rate is less than $20 \mathrm{l} / \mathrm{min}$. However, these values approach the calculated values based upon axial flow with no cross-sectional flow in the case where gas flow rate is larger than $40 \mathrm{l} / \mathrm{min}$.

\section{Conclusion}

The temperature distributions and concentration distributions of reactant gas in a horizontal epitaxial reactor were investigated experimentally and theoretically in the high flow rate range, that is, $Q=10-60$ $l / \mathrm{min}\left(20^{\circ} \mathrm{C}, 1 \mathrm{~atm}, R e=160-1400\right)$, and the following results were obtained.

1) The temperature distributions in the reactor in the case of no axial temperature gradient in the high flow rate range were between the calculated tem- 
perature distributions for spiral flow and the calculated temperature distributions for axial flow with no crosssectional flow. When the flow rate is less than $20 \mathrm{l} / \mathrm{min}$, the temperature distribution is approximately calculated by the fundamental equations in the case of stable spiral flow. However, when the flow rate is larger than $50 \mathrm{l} / \mathrm{min}$, the temperature distribution is approximately calculated by the fundamental equations in the case of axial flow with no cross-sectional flow.

2) The empirical equation for the estimation of gas temperature distributions in the high flow rate range was obtained.

3) The reactant concentration distributions in the reactor had the same tendency as the gas temperature distributions. When the flow rate is less than $20 \mathrm{l} / \mathrm{min}$, the reactant concentration distribution can be approximately calculated by the fundamental equations in the case of spiral flow. However, when the flow rate is larger than $50 \mathrm{l} / \mathrm{min}$, the concentration distribution is calculated from the fundamental equations in the case of axial flow with no cross-sectional flow.

\section{Acknowledgment}

The authors wish to acknowledge the help of Mr. Masaki Nakayama, Mr. Sadao Banba, Mr. Takashi Suwa and Mr. Tetsuya Maruo in taking the experimental data.

\section{Nomenclature}

$\begin{array}{llr}C & =\text { dimensionless concentration of reactant gas } \\ & \left(=c / c_{0}\right) & {[-]} \\ C_{p} & =\text { heat capacity at constant pressure } & {\left[\mathrm{cal} / \mathrm{g} \cdot{ }^{\circ} \mathrm{C}\right]} \\ c & =\text { concentration of reactant gas } & {\left[\mathrm{mol} / \mathrm{cm}^{3}\right]} \\ \mathscr{D} & =\text { molecular diffusivity } & {\left[\mathrm{cm}^{2} / \mathrm{sec}\right]} \\ D_{e} & =\text { equivalent diameter of tube } & {[\mathrm{cm}]} \\ G & =\text { mass velocity of gas } & {\left[\mathrm{g} / \mathrm{cm}^{2} \cdot \mathrm{sec}\right]} \\ G r & =\text { Grashof number }\left(=g \beta H^{3}\left(t_{H}-t_{w}\right) / \nu^{2}\right) & {[-]} \\ g & =\text { gravitational acceleration } & {\left[\mathrm{cm} / \mathrm{sec}^{2}\right]} \\ H & =\text { channel height } & {[\mathrm{cm}]} \\ K & =\text { dimensionless reaction rate constant } & \\ & \left(=k_{r} H / \mathscr{D}\right) & {[-]} \\ k_{r} & = & \text { reaction rate constant } \\ L & = & \text { half-width of channel }\end{array}$

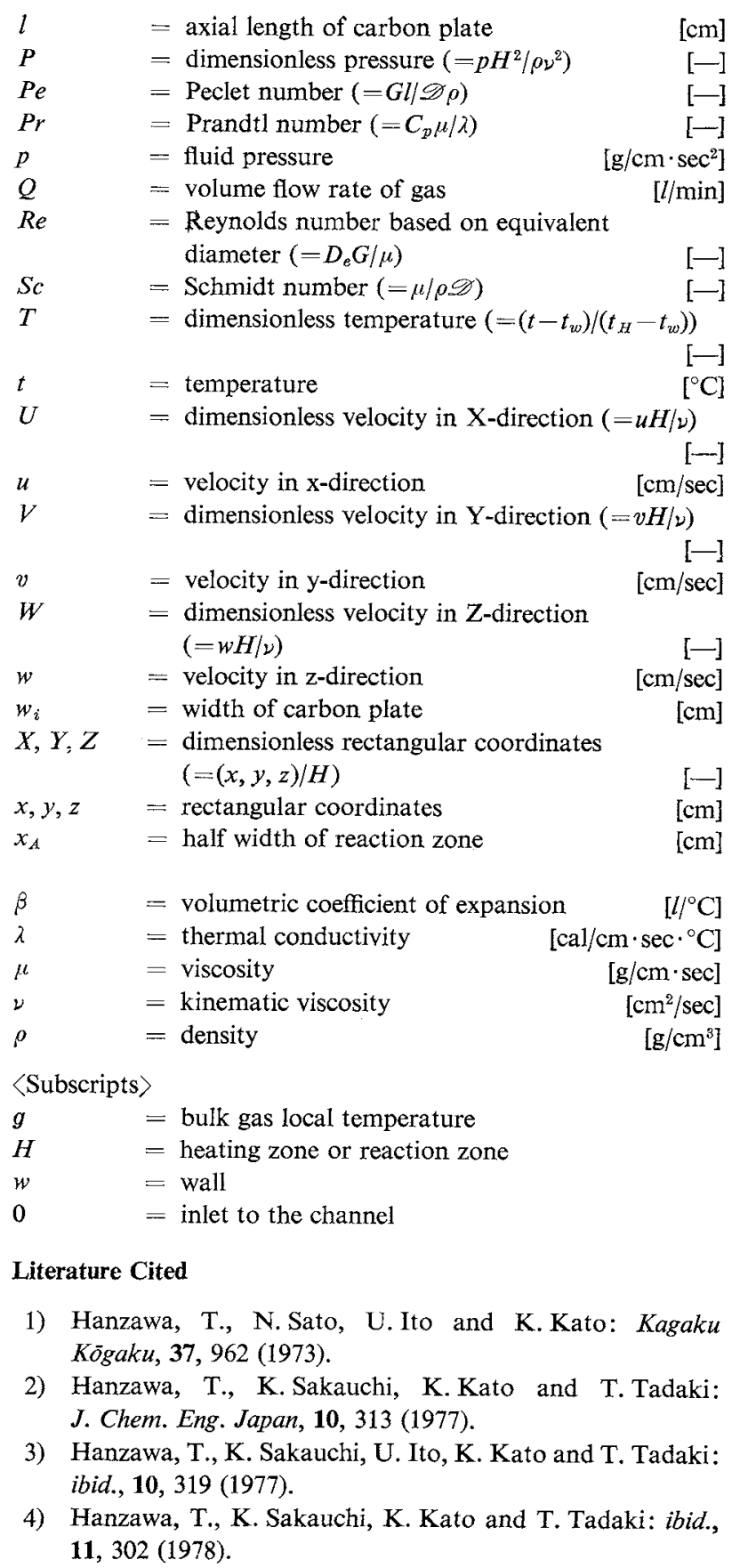

